\title{
A measure of perceived stigma in people with intellectual disability
}

Afia Ali, Andre Strydom, Angela Hassiotis, Rachael Williams and Michael King

\section{Background}

There is a lack of validated instruments measuring perceived stigma in people with intellectual disability.

\section{Aims \\ To develop a valid and reliable self-rated instrument to measure perceived stigma that can be completed by people with mild to moderate intellectual disability.}

\section{Method}

A literature search was used to generate a list of statements. Professionals, individuals with intellectual disability and carers were consulted about the suitability of statements. An instrument was developed containing statements about stigma with accompanying photographs. Test-retest reliability, internal consistency and the factor structure of the instrument were evaluated.

\section{Results}

The instrument was completed by 109 people once and 88 people twice. Items with limited variability in responses and kappa coefficients lower than 0.4 were dropped. Exploratory factor analysis revealed two factors: 'perceived discrimination' (seven items) and 'reaction to discrimination' (four items). One item loaded onto both factors. Cronbach's alpha for the ten-item instrument was 0.84 .

\section{Conclusions}

This instrument will further our understanding of the impact of stigma in people with intellectual disability in clinical and research settings.

\section{Declaration of interest}

None.
Stigma is a 'mark' that distinguishes a person as being deviant, flawed, 'spoiled' or generally undesirable. ${ }^{1}$ A second approach defines stigma as a form of negative social stereotyping or categorisation, where societal attitudes based on social norms and values give rise to stigma. ${ }^{2}$

Intellectual disability (or mental retardation) is defined as a significantly below-average level of intellectual functioning (IQ less than 70) with associated impairments in adaptive functioning (in at least two areas), arising before the age of 18 years. ${ }^{3}$ The UK Valuing People White Paper emphasises the need to improve the lives of people with intellectual disability (also known as learning disability in UK health services) through the promotion of rights, choices, independence and social inclusion, including access to mainstream services. ${ }^{4}$ However, despite these changes, people with intellectual disability continue to remain socially excluded and encounter stigma, prejudice and major barriers that restrict their human rights. ${ }^{5}$

The stigma of intellectual disability is often neglected by health professionals and researchers. Qualitative research has shown that people with intellectual disability are aware of the stigma of their disability and its social consequences. Those who leave state hospitals or family homes to live in the community, or in independent housing, experience abuse and rejection from others in the community and become aware of the stigma associated with institutions such as hospitals or adult centres for people with intellectual disability. They attempt to hide their disability as a way of avoiding stigmatisation. ${ }^{6,7}$

There is currently a lack of valid and reliable instruments measuring stigma in people with intellectual disability. Such an instrument would be useful in identifying the extent and severity of stigma encountered by service users with intellectual disability.

\section{Method}

\section{Ethics}

Ethical approval was obtained to conduct the study in five London boroughs. The study was conducted between February 2006 and
February 2007. Individuals with mild to moderate intellectual disability were approached via three North London intellectual disability services and other voluntary organisations for people with intellectual disability. All participants were given an information sheet and were asked for informed consent.

\section{Development phase and generation of items}

Development work began with a literature search of the databases EMBASE, Medline and PsycINFO in order to identify validated instruments and questions assessing stigma in people with intellectual disability. Search terms included: stigma OR discrimination AND (learning disability OR mental retardation OR intellectual disability). Two published self-report instruments developed for use in school children and students were identified but both have limitations. The first contains ten items grouped under three categories: feeling different, anxiety and 'poor in group concept' (used to describe the low opinion that people with intellectual disability have of others with intellectual disability). ${ }^{8}$ The items were chosen on the basis of face validity and reflected feelings about being 'different', rather than just negative items. The instrument had good internal consistency (Cronbach's alpha $=0.81$ ) but test-retest reliability was not conducted by the authors. In a subsequent study, only two items were found to have adequate test-retest reliability. ${ }^{9}$ The wording of a number of items is also complex, making the items difficult to understand. The second published instrument is the Experience of Stigma Checklist containing 13 items: 8 items describing stigmatising treatment from key figures (parents, teachers, pupils) and 5 items describing non-threatening items. ${ }^{10}$ The internal consistency of the instrument was found to be low (Cronbach's alpha $=0.61$ ) and test-retest reliability was not conducted. Both of these instruments were not developed through consultation with service users or carers, and therefore content validity is questionable. Items from these instruments were not considered suitable for our use owing to the aforementioned problems and our target group being those aged above 18 years. 
Key publications on stigma were examined in order to derive new questions. Forty-seven statements were generated including simplified versions of 20 statements from an instrument that had recently been developed to measure the stigma of mental illness. ${ }^{11}$

\section{Determining content validity}

In order to determine face and content validity of the questionnaire, we consulted a number of professionals in the field of intellectual disability, carers of people with intellectual disability and individuals with mild to moderate intellectual disability. As a first step, we led an open discussion about stigma at a meeting of psychiatrists, speech and language therapists, occupational therapists, nurses and psychologists, and asked for their views on the statements that had been generated from the literature search. The professionals were asked to rate the statements for comprehensibility, relevance and suitability; to make suggestions for further statements; and to give us their views on the most useful format for the instrument. We analysed the responses in order to arrive at a core set of statements, which were made into an accessible format using simple illustrations with the help of a speech and language therapist.

The statements were then piloted at a group meeting at a local day centre of people with intellectual disability who were recruited through an intellectual disability service. Four women and two men aged 25-55 with mild to moderate intellectual disability consented to participate. The 90 -minute group was facilitated by A.A., an accessible information worker and a member of staff from the local day centre. Participants were asked whether they understood the statements, if they had ever perceived or experienced what was described in each statement and whether or not the illustration by each statement was appropriate and informative. We sought their views on possible response formats ('yes/no' answers $v$. three- or five-point scales) and the size and style of the font used.

Three carers participated in a meeting at a day centre for people with intellectual disability in which they rated the coverage and relevance of the statements and whether or not the individual they cared for had ever had such experiences or feelings.

Based on this feedback the instrument was developed into a final set of 21 statements, each with an accompanying photo image to illustrate the theme of each statement and a response format of 'yes' or 'no', which was the preferred choice of the pilot group. The response 'yes' was represented pictorially with a 'thumbs up' and 'no' with a 'thumbs down' symbol (full questionnaire available on request from the authors). The instrument contained descriptions of experiences and feelings of stigma and included negative and positive statements to reduce response set bias. Each statement was displayed in size 14 font and the accompanying image was in colour. Cartoon images and symbols were avoided to ensure clarity. The final format was tested by three volunteers with mild intellectual disability who found the instrument easy to understand and therefore no further changes were made.

\section{Test-retest reliability}

Participants were recruited from a number of different settings including intellectual disability services, out-patient psychiatric clinics, in-patient wards, day centres, social clubs, educational facilities, voluntary organisations for people with intellectual disability (People First, Elfrida) and supported housing schemes. Each participant was asked to complete the instrument on two occasions between 2 and 6 weeks apart. This time period was chosen to balance the possibility that perceived stigma might change over time against the need to avoid recall of previous responses. Although the instrument is self-rated, assistance was provided to participants who had difficulty reading or understanding any of the words. In order to ascertain comprehension of the items, participants were asked to give examples of situations described in the items before selecting an appropriate response.

\section{Additional information}

We collected data on each participant's age, gender, ethnicity, accommodation, attendance at day services and physical disabilities. In order to increase the sample size and to improve the external validity of the questionnaire, people with both intellectual disability and mental illness were also included.

\section{Analysis}

Total stigma scores were obtained for each participant, with higher scores indicating a greater perception of stigma. These were analysed to identify whether there were any differences in the perception of stigma between different socio-demographic and clinical variables. Using the first set of responses to the questionnaire, items with little variation in response $(80 \%$ or more, selecting one answer or the other) were removed as these would differentiate poorly between people with differing experiences of stigma. The test-retest reliability of each item was estimated using the kappa coefficient. Items with coefficients less than 0.4 were removed. To explore the factor structure underlying the remaining statements, we undertook an exploratory dichotomous factor analysis (given each statement has a binary 'yes/no' response) in MPlus using tetrachoric correlations. We used weighted least squares with mean and variance adjustment as the estimation procedure for dichotomous data. We explored orthogonal and oblique rotations of the factors extracted in order to reduce over fitting of the final model. Internal consistency of the final scale (and sub-scales) and whether this improved with any single item removal was estimated using Cronbach's alpha. The correlations of each statement with the total score and the average correlation with other items were analysed.

\section{Results}

\section{Response rates and demographic and clinical data}

A total of 125 people with intellectual disability were approached and 115 agreed to participate. Six individuals were considered unsuitable because they could not give adequate consent, had poor verbal comprehension or difficulties in communication. The remaining 109 (87\%) participants completed the instrument once: $84(77 \%)$ were able to do so with either no assistance or minimal assistance. The remainder, most of whom had moderate intellectual disability, required assistance in clarifying the questions. Thus, a total of 62 women and 47 men, with a mean age of 41 years (range 18-73, s.d.=13.6) participated. Ninety-four individuals had mild intellectual disability and 15 had moderate intellectual disability. Eighty individuals were White (Table 1). Forty people had mental illness, of whom 10 had psychotic disorders, 25 had affective disorders, 2 had an anxiety disorder, 2 had substance misuse disorders and 2 had 'other' disorders.

Five people refused to complete the instrument on the second occasion and a further 16 people could not be contacted or were not available. The only difference between the 109 participants who completed the instrument once and the 88 who completed it twice was that the former were more likely to have a moderate intellectual disability $(P<0.001)$. 


\begin{tabular}{|l|l|}
\hline \multicolumn{2}{|l|}{ Table 1 Socio-demographic data of participants } \\
\hline Demographic characteristic & $n(\%)$ \\
\hline $\begin{array}{l}\text { Ethnicity } \\
\text { White }\end{array}$ & \\
Other & $80(74.4)$ \\
\hline Physically stigmatising conditions & $29(26.6)$ \\
\hline Cerebral palsy & \\
Multiple sclerosis & $1(0.9)$ \\
Genetic syndromes & $1(0.9)$ \\
Mobility problems & $3(2.8)$ \\
\hline Housing & $7(6.4)$ \\
\hline Lives alone & \\
Lives with family & $28(26.2)$ \\
Supported housing & $52(46.8)$ \\
\hline Employment & $29(26.6)$ \\
\hline Unemployed & $81(74.3)$ \\
Voluntary/unpaid work only & $15(13.8)$ \\
Paid work & $13(11.9)$ \\
\hline Use of amenities & \\
Day centre & $37(33.9)$ \\
College/education & $43(39.4)$ \\
\hline a. A total of 12 ethnic groups were represented in the sample. & \\
\hline
\end{tabular}

\section{Distribution of responses}

There were no differences in the total stigma score (mean 6.50, s.d.=3.73) resulting from level of intellectual disability $(P=0.19)$, gender $(P=0.49)$, age $(P=0.70)$, ethnicity $(P=0.51)$, mental health problems $(P=0.20)$, physical disability $(P=0.135)$, employment status $(P=0.57)$, housing $(P=0.19)$, attendance at college $(P=0.58)$ or day centre $(P=0.94)$ in this sample. There were no differences in the responses given to individual items on the questionnaire by participants with mental illness and those without mental illness (Table 2). Three items were ticked 'no' by more than $80 \%$ of the sample (items $3,9,21$ ) and four items were ticked 'yes' by more than $80 \%$ of the participants (items 11,14 ,
16). These items show little variation in response and are less useful in identifying people with differing levels of stigma. These six items have therefore been removed from the analysis (Table 3). The items that were ticked 'yes' by the majority of the sample were 'positive statements' such as 'People like to talk to me' and 'People are nice to me' and may reflect a response set bias.

\section{Test-retest reliability}

Of the remaining 15 items, two had kappa coefficients below 0.4 and were removed (items 2 and 12). The remaining items had kappa coefficients ranging up to 0.71 (Table 3 ).

\section{Factor analysis}

Factor analysis was conducted on the 13 items with acceptable variability in responses and reliability using the 109 responses to the instrument on the first round. Variables with a factor loading of 0.5 or more, where 0.5 is an approximation of the critical value for tetrachoric correlations, ${ }^{12}$ were considered to load significantly onto the respective factor. Two factors were sufficient to explain the intercorrelations among the variables $\left(\chi^{2}=34.51, P=0.12\right)$ (Table 4). The two rotated solutions were very similar in their factor loadings and thus we report the varimax (orthogonal) rotation as it is easier to interpret. Factor 1 (Table 4) consisted mainly of people's experiences of discrimination, such as being bullied or ridiculed and was labelled 'perceived discrimination'. Factor 2 contained statements about people's emotional reactions to stigma such as anger and embarrassment. This factor was labelled 'reaction to discrimination'. Item 20 loaded equally on both factors. The two factors together explained $60 \%$ of the total variance. Items 4, 7 and 13 did not load onto either factor.

\section{Internal consistency}

Cronbach's alpha for the final ten-item instrument (items 1, 5, 6, $8,10,15,17,18,19$ and 20 ) was 0.84 (mean score 5.3, s.d.=3.15).

\begin{tabular}{|c|c|c|c|}
\hline \multirow[b]{2}{*}{ Item/statement } & \multicolumn{2}{|c|}{ 'Yes' responses, $n$ (\%) } & \multirow[b]{2}{*}{$P\left(\chi^{2}\right)$} \\
\hline & Participants without mental illness & Participants with mental illness & \\
\hline 1 People talk down to me & $40(58.0)$ & $25(62.5)$ & 0.64 \\
\hline 2. People think I am not as good as them & $31(44.9)$ & $25(62.5)$ & 0.08 \\
\hline 3. The police has treated me badly & $10(14.5)$ & 9 (22.5) & 0.29 \\
\hline 4. I think I am the same as other people & $55(79.7)$ & $2972.5)$ & 0.34 \\
\hline 5. The way people talk to me makes me angry & $41(59.4)$ & $27(67.5)$ & 0.40 \\
\hline 6. People make me feel embarrassed & $33(47.8)$ & $25(62.5)$ & 0.14 \\
\hline 7. Doctors and nurses have treated me badly & $14(20.3)$ & $9(22.5)$ & 0.79 \\
\hline 8. People on the street make fun of me & $30(43.5)$ & $19(47.5)$ & 0.68 \\
\hline 9. People on the street have hit me & $11(15.9)$ & $8(20.0)$ & 0.59 \\
\hline 10. People on the street look at me in a funny way & $40(58.0)$ & $26(65.0)$ & 0.47 \\
\hline 11. People like to talk to me & $63(91.3)$ & $34(85.0)$ & 0.31 \\
\hline 12. People make fun of my family & $16(23.2)$ & $7(17.5)$ & 0.48 \\
\hline 13. No one bothers me when I use buses, trains or taxis & $52(75.4)$ & $32(80.0)$ & 0.58 \\
\hline 14. I feel welcome in shops, restaurants and pubs & $55(79.7)$ & $33(82.5)$ & 0.72 \\
\hline 15. People laugh at me because of the way I look & $29(42.0)$ & $14(35.0)$ & 0.47 \\
\hline 16. People are nice to me & $64(92.8)$ & $35(87.5)$ & 0.36 \\
\hline 17. People treat me like a child & $28(40.6)$ & $22(55.0)$ & 0.15 \\
\hline 18. I keep away from other people because they are not nice to me & $45(65.2)$ & $28(70)$ & 0.61 \\
\hline 19. People laugh at me because of the way I talk & $25(36.2)$ & $17(42.5)$ & 0.52 \\
\hline 20. I worry about the way people act towards me & $37(53.6)$ & $22(55.0)$ & 0.89 \\
\hline 21. People make fun of me about going to the day centre & $10(14.5)$ & $2(5.0)$ & 0.13 \\
\hline
\end{tabular}




\begin{tabular}{|c|c|c|}
\hline Item/statement & $\kappa$ & $\begin{array}{c}\text { Participants responding } \\
\text { 'yes', \% }\end{array}$ \\
\hline 1. People talk down to me & 0.62 & 60 \\
\hline 2. People think I am not as good as them & 0.39 & 51 \\
\hline 4. I think I am the same as other people & 0.44 & 77 \\
\hline 5. The way people talk to me makes me angry & 0.52 & 62 \\
\hline 6. People make me feel embarrassed & 0.41 & 53 \\
\hline 7. Doctors and nurses have treated me badly & 0.40 & 21 \\
\hline 8. People on the street make fun of me & 0.54 & 45 \\
\hline 10. People on the street look at me in a funny way & 0.61 & 61 \\
\hline 12. People make fun of my family & 0.38 & 21 \\
\hline 13. No one bothers me when I use buses, trains or taxis & 0.62 & 77 \\
\hline 15. People laugh at me because of the way I look & 0.51 & 39 \\
\hline 17. People treat me like a child & 0.58 & 46 \\
\hline 18. I keep away from other people because they are not nice to me & 0.47 & 67 \\
\hline 19. People laugh at me because of the way I talk & 0.71 & 39 \\
\hline 20. I worry about the way people act towards me & 0.41 & 54 \\
\hline
\end{tabular}

No single item deletion led to an improvement in internal consistency. Cronbach's alpha was 0.72 (mean score 3.9, s.d. $=2.2$ ) for the six-item perceived discrimination sub-scale and 0.69 (mean score 2.4, s.d.=1.4) for the four-item reaction to discrimination subscale.

\section{Discussion}

This ten-item self-report instrument for people with mild to moderate intellectual disability has been developed with the detailed input of professionals working with people with intellectual disability, individuals with intellectual disability and carers. It uses an easy-to-understand format with a large font and accompanying photographs illustrating the statements, and takes 5-10 minutes to complete. The 'yes/no' format is readily understood and the majority of respondents were able to complete it with minimal assistance (the final version of the instrument is available as an online supplement to this paper). However, participants with moderate intellectual disability may require additional support. It has an acceptable test-retest reliability and high internal consistency indicating that it can be used as a total score.

\begin{tabular}{|c|c|}
\hline Factor & Factor loadings \\
\hline \multicolumn{2}{|l|}{ Factor 1} \\
\hline 1. People talk down to me & 0.59 \\
\hline 8. People on the street make fun of me & 0.81 \\
\hline 10. People on the street look at me in a funny way & 0.82 \\
\hline 15. People laugh at me because of the way I look & 0.90 \\
\hline 17. People treat me like a child & 0.69 \\
\hline 19. People laugh at me because of the way I talk & 0.70 \\
\hline 20. I worry about the way people act towards me & 0.53 \\
\hline \multicolumn{2}{|l|}{ Factor 2} \\
\hline 5. The way people talk to me makes me angry & 0.79 \\
\hline 6. People make me feel embarrassed & 0.81 \\
\hline $\begin{array}{l}\text { 18. I keep away from other people because they } \\
\text { are not nice to me }\end{array}$ & 0.54 \\
\hline 20. I worry about the way people act towards me & 0.53 \\
\hline
\end{tabular}

The two underlying factors describe useful dimensions of stigma. The 'perceived discrimination' sub-scale examines the reactions of others to people with intellectual disability and includes perceived acts of harassment, ridicule and discrimination. Discrimination is one of the three elements of stigma proposed by Thornicroft et al, who describe discrimination as a 'problem of behaviour.' ${ }^{13}$ The two other elements of stigma are prejudice (problems of attitude) and ignorance (problems of knowledge).

Discrimination is also a central concept of 'enacted stigma. ${ }^{14}$ The extent of discrimination encountered by people with intellectual disability has been highlighted by recent reports from Mencap, a leading organisation in the UK for people with intellectual disability. In a survey of 5000 people, as many as $88 \%$ of people had been bullied over the previous year, with $32 \%$ being bullied on a weekly or daily basis. Forty-seven per cent of people reported verbal abuse and 23\% reported physical violence. ${ }^{15}$ Mencap's report Barriers to Justice suggested that people with intellectual disability were twice as likely to be victims of crime but were not receiving equal and just treatment from the legal process. ${ }^{16}$ Inequalities in healthcare were identified by an investigation conducted by the Disability Rights Commission in the UK and Mencap's Death by Indifference report, which concluded that 'diagnostic over shadowing', where a symptom is misattributed to the disability, and a lack of understanding and adequate training of doctors and nurses, has led to the premature deaths of people with intellectual disability within the National Health Service in the UK. ${ }^{17,18}$

The 'reaction to discrimination' sub-scale examines the emotional reactions of people confronted with stigma. Link \& Phelan argue that emotional reactions are central to the understanding of the behaviour of both the perpetrators and recipients of stigma. ${ }^{19}$ Emotions of anger by the perpetrator may lead to verbal and physical abuse towards the individual with intellectual disability, and emotions of pity and the use of a different tone of voice may signal to the individual that he/she is different. From the perspective of the stigmatised, there may be emotions of shame, embarrassment, alienation, fear and anger. Scheff suggested that shame was a central and particularly distressing feature of stigma. ${ }^{20}$

Response set bias or acquiescence (tendency to say 'yes' to questions regardless of the content) is a potential problem when interviewing people with intellectual disability. ${ }^{21}$ Possible causes 
of acquiescence include the desire to please, suggestibility and lack of understanding due to complex questions. Acquiescence is more likely to occur when using 'yes/no' questions and is inversely related to IQ. ${ }^{22}$ However, Gudjonsson argues that acquiescence is a result of difficult or complex questions rather than a problem of 'yes/no' questions per se. ${ }^{23}$ In our study, no single participant answered 'yes' or 'no' to all the questions, suggesting that acquiescence was low. We have also excluded questions that were answered 'yes' or 'no' by the majority of the participants. One strategy that can be employed to reduce acquiescence is the use of screening questions to exclude participants who are likely to give acquiescent responses. These questions are usually based on nonsense questions and pairs of reverse worded questions but both have limitations: nonsense questions may amuse participants who may say 'yes' to play along and reverse worded questions may produce affirmative answers for both versions as they may conjure up different images or situations. ${ }^{24}$ Screening questions for acquiescence were not used in our study but participants were asked to give examples to illustrate questions.

Questions on time and frequency of perceived stigma were avoided as the notion of time has been found to be a problem when interviewing people with intellectual disability. ${ }^{25}$ We have therefore chosen to look at lifetime experiences of stigma.

The total stigma scores did not vary between sociodemographic and clinical factors in this sample. However, this finding may be explained by our limited sample size. Ethnicity, gender, social class, mental illness and physical disability are factors that may be associated with higher levels of stigma in people with intellectual disability; however, there are few studies looking at this relationship. One qualitative study found that people with intellectual disability were aware of gender and ethnic stereotypes and the disadvantages associated with these. Having an intellectual disability appeared to exacerbate negative gender expectations and detracted from positive ones. ${ }^{26}$ This view is contrary to the view of other researchers who suggest that the experience of disability overrides the experiences of other social identities. ${ }^{27}$ The relationship between socio-demographic factors and stigma therefore requires further investigation. Our finding that perceived stigma was similar in those with and without mental illness suggests that the instrument is suitable for detecting stigma of intellectual disability, irrespective of the presence of mental illness.

\section{Strengths and limitations}

The strengths of the study are that the items and format of the instrument were developed from an extensive search of the literature and consultation with a wide range of people and professionals in the intellectual disability field, including people with intellectual disability themselves. The people with intellectual disability who participated in the field and reliability testing had a range of comorbid problems and were recruited from a number of settings - our sample is therefore comparable with service users with mild to moderate intellectual disability in the UK. The only other existing stigma scales for adults with intellectual disability were not developed with the assistance of individuals with intellectual disability and either had low test-retest reliability or it was not established. ${ }^{8,10}$

One limitation of the instrument is that it attempts to measure 'perceived stigma', which is a subjective measure of stigma and may not reflect actual acts of prejudice or discrimination. However, it is the perception of the discriminatory act rather than the degree of discrimination that is most likely to influence psychological responses and well-being. Therefore, the concept of perceived stigma is valuable. However, perceived stigma can be influenced by a number of factors such as self-esteem, where high self-esteem has been associated with a lower perception of stigma in people with intellectual disability. ${ }^{8,9}$ Likewise, core negative evaluative beliefs about the self are associated with a higher perception of stigma. ${ }^{28}$ These factors will need to be taken into consideration when using the instrument.

Factor analysis has revealed 'perceived discrimination' and 'reaction to discrimination' as being the two underlying components of the scale but we would like to stress that these do not explain all the facets of stigma, which is a complex phenomenon.

The questionnaire will need to be further evaluated in people from ethnic minority groups and in other language or cultural settings to determine its wider applicability. The instrument is not suitable for adults with limited verbal ability or more severe intellectual disability - proxy measures of the experience of stigma will need to be developed for such adults. The instrument is also not appropriate for use in children as it has not been tested in this group. The use of 'yes/no' response formats in instruments for people with intellectual disability has been criticised for high rates of acquiescence and many recommend using 'either/or' questions in preference. ${ }^{21}$ However, such questions are likely to be more complex and difficult to understand and have been shown to have low test-retest reliability. ${ }^{29}$ Although we have used a 'yes/no' format, we have taken steps to reduce acquiescence by carefully developing the items using content validity procedures, using pictures to clarify the items, using negative and positive questions (although all the positive questions were found to have high acquiescence and were therefore removed) and asking people to verify their responses by giving examples. After consultation with experts we decided not to use a 'don't know' response to each item to reduce complexity but we acknowledge that our format has the disadvantage of requiring a definite binary response.

Our sample was sufficiently large to determine the psychometric properties of the instrument, but was relatively small for a factor analysis. However, given there were no published instruments of this type for people with learning disability we had no knowledge of the likely communalities that might guide us in making an estimate of the sample size necessary for conducting a stable factor analysis. ${ }^{30}$ Thus, we stress that this analysis is preliminary and requires replication in other studies. We believe, however, that our results are of interest as it is difficult to recruit large numbers of participants for this kind of development work in this population.

\section{Use of the instrument in research and clinical settings}

This instrument has potential for use in a number of clinical and research settings such as understanding the part played by stigma in utilisation of intellectual disability services and mental health services, adherence to social or medical interventions and service outcomes in people with intellectual disability. It may help to determine the impact of stigma on mental illness and increase awareness of stigma among health professionals so that intellectual disability services are better equipped to support the needs of this vulnerable group.

\section{Further research}

We are now in the process of developing a version of the instrument for carers of people with intellectual disability with the aim of understanding some of the experiences of people with more severe forms of intellectual disability who are not able to communicate their difficulties. The cultural stability of the instrument also needs to be established to assess its utility in other countries or cultural groups. 
Afia Ali, MBBS, MRCPsych, Tower Hamlets Learning Disability Service, Mile End Hospital, London; Andre Strydom, MSc, MBChB, PhD, MRCPsych, Angela Hassiotis, MA, PhD, FRCPsych, Department of Mental Health Sciences, Faculty of Biomedical Sciences University College Medical School (Bloombury campus), London; Rachael Williams, BSC, MSC, Michael King, MD, PhD, FRCP, FRCGP, FRCPsych, Department of Mental Health Sciences, Faculty of Biomedical Sciences, University College Medical School (Hamstead campus), London, UK.

Correspondence: Afia Ali, Tower Hamlets Learning Disability Service Beaumont House, Mile End Hospital, Bancroft Road, London E1 4DG, UK. Email: Afia.Ali@thpct.nhs.uk

First received 14 Sep 2007, final revision 7 Mar 2008, accepted 15 May 2008

\section{References}

1 Jones EE, Farina A, Hastorf AH, Marcus H, Miller DT, Scott RA. Social Stigma: The Psychology of Marked Relationships. Freeman and Company, 1984.

2 Baxter $C$. Investigating stigma as stress in social interactions of parents. J Ment Def Res 1989; 33: 455-65.

3 American Psychiatric Association. Diagnostic and Statistical Manual of Mental Disorders (4th edn) (DSM-IV). APA, 2000.

4 Department of Health. Valuing People: A New Strategy for People with Learning Disability for the 21st Century. Department of Health, 2001. (http://www.archive.official-documents.co.uk).

5 European Union Monitoring and Advocacy Programme. Report on People with Intellectual Disabilities. Open Society Institute, 2004. (http:// www.eumap.org/)

6 Edgerton RB. The Cloak of Incompetence: Stigma in the Lives of the Mentally Retarded. University of California Press, 1967.

7 Jahoda A, Markova I. Coping with social stigma: people with intellectual disabilities moving from institutions and family home. J Intellect Disabil Res 2004; 48: 719-29.

8 Szivos-Bach, SE. Social comparisons, stigma and mainstreaming: the self esteem of young adults with mild mental handicap. Mental Handicap Res 1993; 6: 217-36.

9 Abraham C, Gregory N, Wolf L, Pemberton R. Self esteem, stigma and community participation amongst people with learning difficulties living in the community. J Community Appl Soc Psychol 2002; 12: 430-43.

10 Cooney G, Jahoda A, Gumley A, Knott F. Young people with intellectual disabilities attending mainstream and segregated schooling: perceived stigma, social comparison and future aspirations. J Intellect Disabil Res 2006; 50: 432-44.

11 King $M$, Dinos S, Shaw J, Watson R, Stevens S, Passetti F, weich S, Serfaty M. The Stigma Scale: development of a standardised measure of the stigma of mental illness. Br J Psychiatry 2007; 190: 248-54.
12 Stevens J. Applied Multivariate Statistics for Social Sciences. Lawrence Erlbaum, 1986.

13 Thornicroft G, Rose D, Kassam A, Sartorius N. Stigma: ignorance, prejudice or discrimination? Br J Psychiatry 2007; 190: 192-3

14 Jacoby $A B$. Felt versus enacted stigma: a concept revisited: evidence from a study of people with epilepsy in remission. Soc Sci Med 1994; 38: 269-74

15 Mencap. The Need to Combat Bullying of People with a Learning Disability. Mencap, 2000.

16 Mencap. Barriers to Justice. Mencap, 1997.

17 Mencap. Death by Indifference. Mencap, 2007 (http://www.mencap.org.uk/ case. asp?id=52)

18 Disability Rights Commission. Equal Treatment: Closing the gap. A formal investigation into the physical health inequalities experienced by people with learning disabilities and/or mental health problems. 2006. (http://www.equalityhumanrights.com/Documents/Disability/Formal_ investigations/Health_investigation/Health_FI_part1.pdf

19 Link BG, Phelan JC. Conceptualizing stigma. Annu Rev Sociol 2001, 27 : 363-85.

20 Scheff TJ. Shame in the labelling of mental illness. In Shame: Interpersonal Behaviour, Psychopathology and Culture (eds P Gilbert, B Andrews): 191-205. Oxford University Press, 1998.

21 Finlay WML, Lyons E. Acquiescence in interviews with people who have Mental retardation. Ment Retard 2002; 40: 14-29.

22 Sigelman CK, Budd EC. Pictures as an aid in questioning mentally retarded persons. Rehab Couns Bull 1986; 29: 173-81.

23 Gudjonsson $\mathrm{GH}$. The relationship of intellectual skills to suggestibility, compliance and acquiescence. Pers Individ Diff 1990; 11: 227-31.

24 Rapley M, Antaki C. A conversation analysis of interviews with people who have learning disabilities. Clin Psychol Forum 1996; 84: 17-23.

25 Booth $\mathrm{T}$, Booth $\mathrm{W}$. The use of depth interviewing with vulnerable subjects: Lessons from a research study of parents with learning difficulties. Soc SCi Med 1994; 39: 415-24.

26 McDonald KE, Keys CB, Balcazar FE. Disability, race/ethnicity and gender: themes of cultural oppression, acts of individual resistance. Am J Community Psychol 2007; 39: 145-61.

27 Vernon A. The dialects of multiple identities and the disabled people's movement. Disabil Soc 1999; 14: 385-98.

28 Dagnan D, Waring M. Linking stigma to psychological distress: testing a social-cognitive model of the experience of people with intellectual disabilities. Clin Psychol Psychother 2004; 11: 247-54.

29 Wehmeyer ML. Reliability and acquiescence in the measurement of locus of control with adolescents and adults with mental retardation. Psychol Rep 1994; 75: 527-37.

30 MacCallum RC, Widaman K F, Zhang S, Hong S. Sample size in factor analysis. Psychol Methods 1999; 4: 84-99. 